6. In the third sentence of the last paragraph in the 'Discussion' section:

'However, before that, important questions such as a more molecular basis'

Replace 'a more' with 'the exact'.

7. Use 'TGF- $\beta 1$ ' primers in 'Supplementary table' instead of 'CXCL8'
Forward: 5'-GTGTGGAGCAACATGTGGAACTC TA-3'

Reverse: 5'-TTGGTTCAGCCACTGCCGTA-3'

8. In Figure S2C, change 'CXCL8' to 'TGF- $\beta 1$ '. We were previously confused and used the human data of CXCL8. We apologize for that. Mouse does not have CXCL8. We have now provided the modified Figure S2.

\title{
RETRACTION
}

\section{Requirement for chromatin-remodeling complex in novel tumor suppressor HIC1-mediated transcriptional repression and growth control}

\author{
B Zhang, KJ Chambers, D Leprince, DV Faller and S Wang
}

Oncogene (2011) 30, 4374; doi:10.1038/onc.2011.388

Retraction to: Oncogene (2009) 28, 651-661; doi:10.1038/ onc.2008.419; published online 17 November 2008

The authors wish to retract this article after examination of the publication raised concerns that the primary real-time PCR data, which underlie several of the figures in the article, may not be accurate. The integrity of all of the data presented therefore cannot be assured. 